\title{
Succès, persistance et spécificité de management par projet dans la création et la culture
}

Project management success, persistence and specificity in creation and culture

\section{Paul Rasse}

\section{(2) OpenEdition}

\section{Journals}

Édition électronique

URL : http://journals.openedition.org/communicationorganisation/4921

DOI : 10.4000/communicationorganisation.4921

ISSN : 1775-3546

Éditeur

Presses universitaires de Bordeaux

Édition imprimée

Date de publication : 1 juin 2015

Pagination : 131-140

ISSN : $1168-5549$

Référence électronique

Paul Rasse, «Succès, persistance et spécificité de management par projet dans la création et la culture », Communication et organisation [En ligne], 47 | 2015, mis en ligne le 01 juin 2018, consulté le 01 mai 2019. URL : http://journals.openedition.org/communicationorganisation/4921 ; DOI : 10.4000/ communicationorganisation.4921 


\title{
Succès, persistance et spécificité de management par projet dans la création et la culture
}

\author{
Poul Rosse
}

Le capitalisme doit son succès planétaire à sa capacité à conjuguer certaines formes de liberté et de créativité comme moteur de l'innovation et facteur de la compétitivité des entreprises, avec, paradoxalement, l'extension des processus de contrôle indispensables à l'organisation de la production et de la consommation à l'échelle industrielle. Nous nous intéresserons ici plus particulièrement au concept de projet qui est sans doute la cheville ouvrière d'un dispositif visant la créativité, à susciter les initiatives autant qu'à les surveiller (Rasse 2013, Rasse 2010, Rasse 2006). Dans une seconde partie nous verrons en quoi le champ de la culture et la vie d'artiste illustrent bien les mutations du monde à l'œuvre.

\section{Un contexte historique, technologique et managérial favorable}

L'informatique couplée à la télématique, en rendant possible la gestion et la circulation de masses considérables d'informations, aura permis non seulement de casser les usines géantes de la période fordienne devenues ingérables, mais encore d'approfondir et de redistribuer la division du travail à l'échelle mondiale en fonction des opportunités du moment, d'accès à une main-d'œuvre aux plus bas coûts possibles, tout en maintenant, et même, en améliorant le contrôle des travailleurs et des processus de fabrication. Les régions les plus avancées où étaient implantés les centres de décisions ont ainsi pu se débarrasser du travail parcellisé, à la chaîne, critiqué et dénoncé comme aliénant, pour ne conserver que la partie la plus créative et innovante des processus : les choix stratégiques, la conception, la recherche, le développement,

1 Paul Rasse est Professeur des Universités, à l'Université Nice Sophia - Antipolis. Directeur du laboratoire de recherche en sciences de l'information et de la communication "I3M" (information, milieux, médias, médiation), regroupant une cinquantaine enseignants chercheurs et autant de doctorants des Universités Nice - Sophia Antipolis et du Sud Toulon Var (EA n 3820). Il est aussi Directeur du Master 2 Évènementiel, médiation et ingénierie culturelle et 1er Vice Président de la SFSIC (Société française des sciences de l’information et de la communication). Il a publié une quinzaine d'ouvrages comme auteur ou codirecteur et de nombreux articles scientifiques, sur l'anthropologie de la communication, la culture et les musées, les mutations de la société hypermoderne ; rasse@unice.fr 
le financement, le marketing, la publicité et la distribution. Dans le même temps, en s’inspirant des méthodes managériales japonaises, les industriels ont porté leurs efforts sur les attentes de la clientèle pour améliorer la fiabilité des biens de consommation. Les nouvelles méthodes managériales, après avoir rendu perméable la séparation taylorienne entre conception et exécution, s'attaquent maintenant aussi à la césure production/usage, jusqu'à associer les consommateurs dans des processus de co-conception (Proulx, Coutant). Le contrôle de la qualité, facilité par l'informatisation des spécifications, les y a largement aidés, tandis que la globalisation du marché planétaire offrait aux consommateurs la possibilité d'accéder à des biens matériels et culturels tout aussi standardisés, mais sans cesse renouvelés et, en apparence au moins, abondamment diversifiés. Et cela a permis à "l'esprit du capitalisme ", pour reprendre l'expression de Boltanski et Chiapello, de recycler les aspirations et les critiques soixante-huitardes à l'égard de l'aliénation du travail autant que de la consommation de masse (Boltanski - Chiapello, Querien).

Dans les pays développés, les méthodes managériales tayloriennes ont été progressivement abandonnées pour libérer les initiatives et donner à chacun plus de liberté dans son espace de travail, de façon qu'il puisse révéler ses potentialités, y exprimer ses talents et contribuer aux processus d'intelligence collective nécessaires à la compétitivité des entreprises, et notamment des multinationales devenues centres de décision, de recherche et développement, d'organisation et de contrôle du travail à l'échelle mondiale. Sans doute est-ce pour cela que dans les années 1990 le concept de projet s'est imposé un peu partout dans les entreprises comme dans les services et la culture. Sans doute aussi, est-ce parce qu'il correspondait parfaitement à la nouvelle donne qu'il a perduré parmi les technologies managériales des dirigeants pourtant toujours avides de nouveautés, alors que dans le même temps bien d'autres méthodes ont périclité, aussi vite qu'elles avaient suscité à leur époque de véritables engouements.

Le succès, la vitalité et la persistance du concept de projet tiennent déjà à ce qu'il rend visibles les processus de création et d'innovation, jusque-là très informels et imperceptibles, noyés qu'ils étaient dans les pratiques ordinaires, la masse des activités quotidiennes nécessaires à l'organisation de la production. Dans le diagramme des activités de l'entreprise, il met en évidence les pics d'innovation créative pour les susciter et les soutenir, donc pour leur accorder l'attention et les moyens nécessaires, en même temps que pour les sélectionner et les contrôler. Il permet de créer des équipes pluridisciplinaires organisées et télé dirigées par les objectifs innovants assignés au projet, en tant qu'évaluées c'est-à-dire contrôlées relativement à ces mêmes objectifs.

Outre les fonctions classiques de mise en forme et d'explication d'une intention, de préparation et de planification des façons d'y parvenir, le projet devient le moyen de recomposer, dans le même espace symbolique, des actions entreprises sur des lieux et en des temps de plus en plus dispersés. De 
façon que chacun, s'il pousse ses pions sur un échiquier virtuel, ait toujours en mémoire qu'il inscrit ses coups dans un jeu collectif global.

- Il permet de coordonner le travail des différents acteurs disséminés un peu partout, quand ils ne sont pas devenus nomades, de rendre visible à tous leur progression et au final de l'évaluer.

- Il rappelle en permanence comment les tâches éparpillées et désynchronisées des uns et des autres, que chacun fait en son temps, doivent s'imbriquer dans un temps collectif, dans le planning de suivi du projet.

Sur le plan individuel, le management par projet parvient à conférer du sens et de la cohérence au travail en miettes des cadres absorbés par des activités qui les mettent tour à tour en relation avec d'autres univers, d'autres travailleurs, ou des données virtuelles dispersées aux quatre coins du monde, dans des espaces immatériels et désynchronisés. Combien de temps chacun passet-il maintenant, seul dans la nuit scintillante du Web, à communiquer par mail, serveurs et banques de données interposées avec des acteurs anonymes sans autre visage que le design et l'ergonomie des pages d'écran, et dont la personnalité s'explique au mieux sous la forme d'émoticônes standardisées et de quelques phrases laconiques en forme de mails, tombant par centaines chaque jour dans les boîtes aux lettres virtuelles, où s'accumulent les sujets, les objets, les auteurs..., et que chacun explore en son temps? Au bout, face à cette fragmentation des collectifs de travail, des existences singulières et des tâches personnelles, seul encore le projet est en mesure de rassembler les micro contributions ultra spécialisées des uns et des autres et de les regrouper, de les réarticuler entre elles pour leur conférer du sens, de la signification et de la réalité.

Si le management par projet permet de mobiliser l'intelligence de tous et aux individus d'avoir le sentiment de se réaliser, d'exprimer ses talents, de cultiver son authenticité, il a pour contrepartie un stress croissant des sujets écartelés en de multiples activités individuelles et collectives, toujours à voyager physiquement ou virtuellement, pour aller de l'une à l'autre, toujours à explorer de nouveaux espaces de relation et à recomposer leur environnement. Les plus dynamiques, les mieux préparés, les bien fournis en capital social, ayant accès à des réseaux de sociabilité opérant, s'y épanouissent. Ambitieux, mobilisés, offensifs, ils peuvent raisonnablement espérer progresser. Mais malheur aux moins forts, aux fatigués, aux vieillissants accrochés à des repères qui n'existent déjà plus (Boltanski - Chiapello : 168). Dès qu'ils ne sont plus dans le mouvement, ils sont éjectés, marginalisés, exclus du cœur bouillonnant où tout se joue; alors que d'autres balises s'allument plus loin, qu'ils ne voient pas et offrent des opportunités dont ils ne peuvent plus se saisir. L'entreprise les maintient parfois dans des postes moins exposés, généralement elle offre à tous ses employés un minimum de sécurité, la possibilité d'attendre, de se repositionner entre l'achèvement d'un projet et le début d'un autre ; mais qu'advient-il des indépendants, des contractuels, des intermittents, de 
tous ceux dont elle s'efforce d'externaliser les contributions pour gagner en flexibilité ?

Les technologies de l'information et de la communication ont rendu possible la dispersion des entreprises en de petites unités, sensibles, souples, réactives, immergées dans le tissu social des pays les plus avancés et les plus puissants, à l'affût des aspirations et des transformations du corps social, pour les anticiper, les accélérer, surfer sur elles, imaginer et dégager des espaces de rentabilisation possible du capital investi. Car les innovations sont à la fois inductrices de transformation des modes de vie et en prise sur la société pour pouvoir les poursuivre. Elles peuvent associer les usagers dans les processus de co-conception, ou encore analyser la myriade de traces laissées par chacun dans ses activités de vie quotidienne. Mais plus en amont et indirectement encore, on peut imaginer que la capacité d'innovation des entreprises dépend de la société dans laquelle elles se développent. Pour garder leur avance, pour créer, inventer, susciter de nouvelles habitudes, des modes et des tendances, il faut que la cité qu'elles habitent, où elles sont implantées, se transforme elle aussi, il faut qu'elle s'approprie les innovations et les utilise pour se réinventer de façon permanente.

Dès lors on imagine combien la présence de toutes sortes d'artistes, de créateurs, de réalisateurs, de critiques, contribue fortement à cette dynamique. Car, ainsi que le dit Menger, "ils mettent l'accent sur les valeurs cardinales d'innovation, de connaissances, d'apprentissage et de motivation pour désigner les secteurs mus par le ressort de la créativité (les arts et les sciences) et, par contiguïté, ceux qui le sont avec des objectifs appliqués (l'éducation, la recherche et le développement technologiques, la communication et la publicité) comme des réservoirs de connaissance, de préceptes, de recettes, d'outils transférables, ou opposables, à l'ensemble des sphères de production " (Menger $2002: 22$ ).

\section{Caractéristiques managériales du monde de la culture}

Le monde de la culture illustre bien cette tendance de la société contemporaine que Boltanski et Chiapello appellent " la cité par projet " c'est-à-dire une société où le modèle de management et de fonctionnement par projet s'est généralisé. Dans un monde connexionniste, expliquent-ils, la distinction entre vie privée et vie professionnelle tend à s'effacer... À la différence de la période précédente celle de l'industrialisation où « l'activité se confondait avec le travail et où les actifs étaient par excellence ceux qui disposaient d'un travail salarié stable et productif ", l'activité dans la cité par projet (caractéristique de la période actuelle) «surmonte les oppositions du travail et du non travail, du stable et de l'instable, du salariat et du nonsalariat, de l'intéressement et du bénévolat, de ce qui est évaluable en terme de productivité et de ce qui n'étant pas mesurable, échappe à toute évaluation comptable»(Boltanski, Chiapello : 166). L'activité par excellence consiste 
à entretenir un réseau et à y intervenir par séquence, à le réactualiser en se donnant sans cesse des occasions d'y participer (Menger2002: 40). Autrement dit, pour être au monde et rester dans le coup, il est indispensable que chaque artiste contribue à une multitude de projets, professionnels ou non, sur des statuts parfois très différents, salariés ou bénévoles, mais aussi qu'il ait les siens pour affirmer son identité, sa personnalité. Dans tous les cas, la capacité à ressaisir et à formuler des projets devient un atout indispensable, d'autant plus indispensable que l'on est nouveau venu dans ce jeu, avec une réputation encore à construire (Rasse 2008).

Les concepts managériaux ont été jusque-là forgés dans des entreprises puissantes, mais surtout pérennes, stables, organisées pour durer. Le champ de la culture, lui, fonctionne autrement, contraint qu'il est de se renouveler sans cesse ; dans une grande précarité des moyens disponibles, il a dû inventer de nouvelles façons de procéder qui en définitive correspondent davantage aux nouveaux univers d'un monde plus complexe, ouvert, structuré en réseaux, dont les mutations vont en s'accélérant (Benghozi : 72). Il est essentiellement constitué de toutes petites entreprises créatives publiques ou privées, parfois informelles, employant des travailleurs aux statuts et aux revenus extrêmement précaires. Dans l'entreprise, où les prises de décisions sont encore très hiérarchisées, quand la direction décide de lancer telle ou telle opération, elle lui accorde forcément les moyens nécessaires. L'effort des responsables de projets porte alors sur la réalisation, la planification, la gestion et le management. En effet, il s'agit de coordonner le travail de dizaines et, pour les grands projets, de milliers de personnes, parfois dispersées sur la planète entière. À l'inverse, dans le monde de la culture, les initiatives viennent souvent d'en bas, des porteurs de projets qui n'ont pas les ressources nécessaires et sollicitent les moyens matériels et financiers auprès des décideurs, dont la caractéristique est d'être dispersés et déjà très convoités. Avant de mettre en œuvre un projet, la première étape, la plus difficile, parce qu'elle se déroule généralement dans un univers très concurrentiel, est de convaincre les financeurs d'accorder au projet les moyens nécessaires. Si bien que là, plus que nulle part ailleurs, un projet commence par sa formulation et sa négociation avec les partenaires potentiels.

Longtemps, le monde de la culture a été dominé par «le fait du Prince » hérité d'une conception aristocratique, ancienne, des politiques culturelles, qui voulait que quelques personnes seulement, aient à décider de ce qui était bon ou mauvais, beau ou laid, des dépenses somptuaires qu'il convenait d'entreprendre pour le prestige du Roi qui imposait à tous ses goûts. Et cela a perduré bien après les révolutions ; la culture étant justement le dernier lieu où les aristocraties se recomposaient, où les élites se reconnaissaient et se distinguaient (Rasse 2011). Et cela continue sans doute encore, par bien des aspects et dans bien des domaines. En même temps, le paysage se transforme pour faire place à un univers connexionniste en perpétuelle reconfiguration, 
qui bouscule la donne et substitue au pouvoir pyramidal, la force des relations au sein de réseaux hiérarchisés et stratifiés.

En période de stagnation ou de faible progression des budgets alloués à la culture, même les plus puissants n'ont plus les moyens de décider seuls de l'édification d'une institution, de la réalisation d'un événement d'envergure ou de la carrière d'un artiste. Les réalisations se font en croisant les financements, publics ou privés au plan local, national ou international, en mobilisant les moyens d'autres institutions déjà existantes, en rassemblant des équipes de professionnels détachés, de bénévoles plus ou moins disponibles, d'experts à temps partagé... bref, en puisant ici et là des budgets, des forces, des éléments qui sont généralement dispersés et par ailleurs déjà fortement sollicités pour d'autres activités. Et cela contribue, pour le moins, à la diffusion de la culture dans le corps social, à en légitimer les dépenses et à rassurer les financeurs, qui de la sorte partagent les risques. Mais au final, ne résistent que les projets les plus convaincants, qui s'imposent à la raison de tous, et donnent à chacun des participants le sentiment qu'il va progresser en s'y associant d'une façon ou d'une autre. Aussi le projet doit-il être non seulement bon, prestigieux, ambitieux, innovant, mais encore évident, c'est-à-dire en même temps clair, explicite, transparent et faire preuve de réalisme. Il doit enthousiasmer, mais aussi rassurer les partenaires, et pour cela donner l'impression que chaque étape a été soigneusement envisagée et quantifiée, que les coûts et les difficultés n’ont pas été sous-estimés...

Quant aux artistes ou aux réalisateurs, ils doivent sans cesse passer d'un projet à l'autre, anticiper, en imaginer de nouveaux, les préparer, les défendre, chercher les moyens nécessaires, convaincre les décideurs déjà tellement sollicités... Et pour cela il faut du temps, pour la conception et la négociation des projets bien sûr, mais encore pour les castings, pour assister aux séances de sélection et pour recommencer quand on perd. Et puis il faut du temps encore pour maintenir son employabilité, pour rester dans le circuit, les canaux interconnectés du réseau dirait-on maintenant. Pour se maintenir et pouvoir se renouveler, il faut se tenir informé, être à l'affût des bons plans, il faut multiplier les connections en croisant ses appartenances, de façon à être toujours dans l'air du temps, en avance sur les autres, de façon surtout à minimiser les risques de s'échouer, de se perdre, quand un contrat se termine ou que pour une raison ou une autre les réseaux toujours en recomposition se défont, se tarissent et disparaissent, tandis que d'autres balises s'allument déjà, plus loin, qui offrent des opportunités, à condition de les voir et d'avoir encore un minimum de forces à y consacrer...

Certains acteurs du paysage culturel qui participent à ces projets ont un statut de salarié permanent parce qu'ils sont employés d'un théâtre, d'une agence ou d'une collectivité, qu'ils sont permanents d'un festival ou d'une compagnie, enseignant d'une école d'art ou d'un conservatoire, professeur d'arts plastiques ou de musique dans un lycée, quand d'autres encore émargent 
sur des statuts, plus ou moins bancals, mais qui leur assurent un minimum de garanties et de stabilité pendant les périodes d'intermittence. Les statistiques le confirment, le statut d'indépendant demeure une caractéristique forte de l'emploi dans les professions culturelles : un tiers des actifs y exercent leur métier sous statut non-salarié, soit près de trois fois plus que la population active (Gouyo - Patureau 2014). Le travail à temps partiel a également progressé pour concerner un quart des emplois de ce champ en 2011. Enfin, $46 \%$ des salariés des professions culturelles travaillent en horaires variables d'une semaine sur l'autre, et un quart d'entre eux travaillent le soir. À ceuxlà s'ajoutent tous ceux qui n'ont pas accès à un emploi officiel, notamment les stagiaires et les bénévoles. Ainsi on évalue que sur 267000 associations culturelles recensées en France, 35100 d'entres elles emploient au moins un salarié, et que l'activité de toutes les autres repose exclusivement sur la participation bénévole (Deroin 2014). En dépit de ces difficultés le nombre de postulants ne cesse de croître. Ainsi, pour l'année universitaire 2012-2013, la centaine d'établissements relevant du ministère de la Culture a accueilli près de 35000 étudiants, auxquels s'ajoutaient encore les étudiants dans les autres établissements d'enseignement supérieur artistique et culturel estimé à 26000 (Les établissements ... 2015). Si l'on part du principe que ces études durent environ cinq ans, cela fait chaque année 12000 nouveaux artistes diplômés, entrant sur le marché du travail auxquels on pourrait encore ajouter des dizaines de milliers d'étudiants venus de la communication, de la mode, du design, de l'informatique, des réseaux, etc. Ne faut-il pas y voir une richesse pour la société tout entière, le signe d'une dynamique à l'œuvre pour sa transformation, d'un enthousiasme de sa jeunesse pour des activités créatives, de réalisation et d'expression personnelle autant que collectives, qui la renouvelle et la réinvente en permanence pour la maintenir à l'avantgarde des mutations du monde. Et cela en dépit des difficultés et des risques que tous connaissent et que tout le monde leur rabâche inlassablement, car les métiers les plus créatifs ont pour caractéristiques de réserver $80 \%$ des rétributions monétaires ou symboliques mises en circulation à $20 \%$ des individus (Menger 2014 : paragraphe 16). Sans doute, nombre d'entre eux espèrent-ils, secrètement, au début au moins, sortir par le haut, accéder à la notoriété, connaître le succès et toucher les revenus qui vont avec. Mais la plupart ne se font pas, ou plus trop d'illusions ; ils savent que bien des galères et nombre de difficultés les attendent, pour des conditions de sécurité de l'emploi et de rémunération minimales... Pour tous, plus qu'ailleurs encore, le projet constitue un outil essentiel pour formuler et exprimer leurs ambitions, leurs intuitions, leurs perspectives...

Dans un monde connexionniste nous disent Boltanski et Chiapello, tout dépend de la mobilité, de la capacité circuler, et pour cela à se fondre dans la masse, à perdre ses aspérités, à se liquéfier, et en même temps pour exister, pour séduire et intéresser les autres il faut se démarquer, affirmer sa personnalité 
(Boltanski, Chiapello 2004 : 561). Une carrière d'artiste réussie, explique Menger, passe par une mobilité ascendante au sein d'un monde stratifié de réseaux d'interconnaissances et de collaborations récurrentes (Menger 2002 : 40 et suivantes). La dynamique de projet fait et défait les équipes, au fur et à mesure des réalisations. Les groupes, ajoute-t-il, se cooptent, ont tendance à se recruter et à s'appareiller par niveaux d'acteurs, les plus réputés allant ensemble, avec plus de moyens et de facilités, devant d'autres qui le sont moins, mais peuvent espérer grimper à leur tour. Car rien n'est plus inégalitaire, conclut-il : « que la logique d'appariement par niveaux de qualité et de réputation... Il dessine un chemin de crête étroit entre la consolidation des rentes réputationnelles et la contestabilité permanente de ces réputations par les nouveaux arrivants. » (Menger 2002 : 47) Mais pour cela, il faut durer, il faut pouvoir s'investir à fond dans des projets et en même temps rester disponible pour d'autres opportunités, pouvoir nouer des relations avec de nouveaux réseaux, qui offriront plus tard d'autres possibilités.

\section{Au sein de la société de projet, vers davantage de solidarité}

Les mutations de la société par projets que les artistes explorent et contribuent activement à façonner devraient avoir pour contrepartie, que l'État, les collectivités, les employeurs garantissent à ces nouvelles formes d'activité, un minimum de sécurité, de protection contre le chômage et les accidents de la vie. Dans un monde que les nouvelles technologies de communication rendent toujours plus mobiles, obligeant à toujours plus de flexibilité et de précarité, il devient indispensable d'inventer de nouvelles formes de solidarités, car elles conditionnent la créativité et la capacité d'une société à se renouveler. Ainsi que le rappelle Menger dans sa leçon inaugurale au collège de France «Pour que les individus, les communautés professionnelles et les organisations puissent s'engager dans le jeu risqué de l'invention créatrice, ils doivent disposer de moyens efficaces pour gérer les incertitudes d'emploi et de carrière. Sans ces moyens, les bénéfices collectifs d'une économie de la différenciation et du risque seraient introuvables » (Menger 2014 : paragraphe 100). Il faut que d'une façon ou d'une autre la société puisse garantir à chacun, plus que la reproduction de sa force de travail, le maintien de son employabilité au meilleur niveau, et donc, la possibilité d'améliorer sa formation, son information, de participer aux réseaux, de contribuer à l'élaboration de nouveaux projets même quand les personnes n'ont plus d'employeur direct. C'est ce que prévoit le régime des intermittents ; malheureusement au lieu de s'étendre à de nouveaux domaines d'activité, il tend plutôt à se réduire comme une peau de chagrin. Le succès du capitalisme a facilité l'essor de la pensée ultralibérale qui met en question les formes de solidarité violemment, laborieusement, patiemment construites au XX siècle, et nous ramène par bien aspects au XIX . Bien sûr, il faut réguler, évaluer, réglementer les conditions d'accès à ces formes de solidarité, tout en évitant 
de stigmatiser les bénéficiaires, pour en faire un droit collectif. Les générations précédentes l'ont fait avec le projet de la modernité, notamment au sortir de la seconde guerre mondiale dans une France et une Europe en ruine, et bien avant encore au cours du siècle dernier, alors que cela paraissait inconcevable et inadmissible à beaucoup. Il s'agit de faire en sorte que le meilleur de ce monde-là ne demeure pas réservé aux élites et aux classes moyennes aisées, en ce sens où elles seules disposent d'un capital économique, social et culturel suffisant pour être à l'aise dans ces nouveaux univers et profiter au mieux des possibilités de réalisation personnelle qu'ils offrent, alors que les plus pauvres, comme, toujours, en paient le prix fort en termes de dégradation et de précarisation de leurs conditions de vie et de travail.

\section{BIBLIOGRAPHIE}

BENGHOZI Pierre-Jean, La gestion de projet dans le secteur culturel, Hermès, $\mathrm{n}^{\circ} 44$, 2004, p. 71-78.

BOLTANSKI Luc, Chiapello ève, Le nouvel esprit du capitalisme, Gallimard, 2004, $843 \mathrm{p}$.

COUTANT Alexandre, "Les approches sociotechniques dans la sociologie des usages en SIC ", Revue française des sciences de l'information et de la communication [En ligne] Pelissier Nicolas, Badillot Patrick-Yves (sld), Usages et usagers de l'information à l'ère numérique, $\mathrm{n}^{\circ}$ 6, 2015, mis en ligne le $1^{\text {er }}$ janvier 2015, consulté le 18 février 2015. URL : http://rfsic.revues.org/1271.

Les établissements de l'enseignement supérieur Culture, http://www. culturecommunication.gouv.fr/Politiques-ministerielles/Etudes-et-statistiques/Lesstatistiques-culturelles/Les-etablissements-de-1-enseignement-superieur-Culture, consulté le 21/02/2015

MENGER Pierre Michel, Portrait de l'artiste en travailleur. Métamorphoses du capitalisme, Paris, Seuil, 2002, 97 p.

MENGER Pierre-Michel, La différence, la concurrence et la disproportion. Sociologie du travail créateur, Leçon inaugurale prononcée le jeudi 9 janvier 2014, éd. Collège de France, 2014.

PROULX Serge, "La sociologie des usages, et après ? ", Revue française des sciences de l'information et de la communication [En ligne] Pelissier Nicolas, Badillot Patrick-Yves (sld), Usages et usagers de l'information à l'ère numérique, $\mathrm{n}^{\circ}$ 6, 2015, mis en ligne le

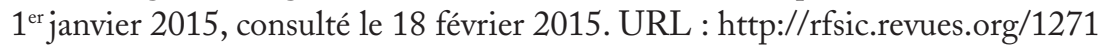

QUERIEN Anne, Le capitalisme à la sauce artiste retour sur le nouvel esprit du capitalisme, Multitudes, $\mathrm{n}^{\circ}$ 15, hiver 2004, p. 251-261.

RASSE Paul, Approche anthropologique de la crise des intermittents, in Pelissier Nicolas et Lacroix Céline, (sld), Les intermittents du spectacle, l'Harmattan, 2008, p. 7-22.

RASSE Paul, Conception, management et communication de projets culturels, Grenoble, Territoriales éditions, réédition revue et augmentée, 2013, 104 p.

RASSE Paul, Le musée protagoniste de l'art contemporain, Hermès, n 61, Ed. CNRS, 2011, p. 76-83 


\section{$C \& O n^{\circ} 47$}

RASSE Paul (sld), La mondialisation de la communication, coll. Les essentiels d'Hermès, Ed. CNRS, 2010. Rasse Paul, La rencontre des mondes : Diversité culturelle et communication, Paris, Armand Colin, collection sociétales, 2006, 226 p.

SOTTO Marie-Françoise, Les établissements de l'enseignement supérieur Culture, année universitaire 2010-2011 Cultures en chiffres - DEPS, 2012-1.

SOURCES : Deroin Valérie, Emploi, bénévolat et financement des associations culturelles Cultures en chiffres - DEPS, 2014-1

SOURCES : Gouyon Marie, Patureau Frédérique, Vingt ans d'évolution de l'emploi dans les professions culturelles, 1991-2011, Cultures en chiffres - DEPS, 2014-6

SOURCES : Les établissements de l'enseignement supérieur Culture, http://www. culturecommunication.gouv.fr/Politiques-ministerielles/Etudes-et-statistiques/Lesstatistiques-culturelles/Les-etablissements-de-1-enseignement-superieur-Culture, consulté le 21/02/2015

Résumé : Le succès persistant du concept de projet comme pivot des technologies managériales, tient à sa capacité à développer la créativité, à susciter les initiatives autant qu'à les surveiller, les contrôler et les évaluer. Le champ de la culture et la vie d'artiste illustrent bien les mutations à l'œuvre dans le travail, où la passion de la création se conjugue avec la précarisation des conditions d'existence.

Mots-clés : le concept de projet, technologies managériales, le projet culturel, vie d'artiste, précarité.

Abstract: The persistent success of the concept of project as a key role of managerial technologies is due to its capacity to develop creativity, to generate initiatives as well as to monitor, control and evaluate them. The cultural field and the artist's life perfectly illustrate the changes which occur in the working area, where passion of creating is combined with the precariousness of living conditions.

Keywords : the concept of project, managerial technologies, cultural project, life as an artist, precariousness. 